\title{
Revista Médica de Chile: Nuevo miembro del "International Committee of Medical Journal Editors"
}

\author{
HUMBERTO REYES B. ${ }^{1}$
}

\section{Revista Médica de Chile: A new member in the "International Committee of Medical Journal Editors"}

\begin{abstract}
After a worldwide call for applications that took place in 2009, two medical journals were selected to become new members of the "International Committee of Medical Journal Editors (ICMJE)": Chinese Medical Journal and Revista Médica de Chile. Both Editors: Getu Zhaori, MD, and Humberto Reyes, MD, respectively, attended the ICMJE Meeting, 12-14 April 2010, in Queenstown, New Zealand. The meeting agenda included several topics that had been studied in advance by the attendants: editors or deputy editors of the 14 journals integrating this Committee plus a representative for the U.S. National Library of Medicine and another one for the World Association of Medical Journal Editors (WAME). The Committee agreed in new recommendations tending to safeguard the integrity and transparency of every manuscript published in all journals that adhere to the "Uniform Requirements for Manuscripts Submitted to Biomedical Journals (URM)". These recommendations will be published shortly in ICMJE member journals. An important issue discussed refers to improvements in the "ICMJE Uniform Disclosure Form for Potential Conflicts of Interest", that had some changes, a glossary of terms will be attached to it and the Instructions will be accompanied by translations into the official WHO languages as well as other languages used by ICMJE member journals. For our journal it is an honor and a great responsibility to become a member of this highly qualified Committee, the only journal published in Spanish and the first one from Latin America.

(Rev Med Chile 2010; 138: 665-668).
\end{abstract}

Key words: Committee membership; Editorial policies; Journalism, medical.

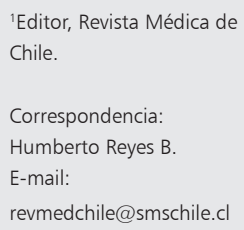

E n 1978, diecinueve editores de revistas médicas de prestigio reconocido, de Norteamérica y Europa, autodesignados "International Steering Committee", se reunieron en Vancouver, Canadá, para generar un documento con recomendaciones sobre el formato que deberían tener los manuscritos al someterse a publicación en revistas biomédicas. Dieron una atención particular a las referencias bibliográficas, para las cuales adoptaron el estilo exigido por la National Library of Medicine, de los EE UU de NA (en Index Medicus y, posteriormente, en MEDLINE/PubMed). El documento se tituló "Uniform Requirements for $\mathrm{Ma}$ nuscripts Submitted to Biomedical Journals (URM)" y se publicó en 1979 en revistas representadas en ese Comité, invitando a todas las revistas médicas a adoptar sus proposiciones ${ }^{1}$. El Comité continuó reuniéndose periódicamente para revisar el documento, modificándolo y ampliándolo según las observaciones que recibieron desde distintos ámbitos, particularmente de editores y autores de manuscritos. En años posteriores hubo cambios en 
la composición del Comité, incorporándose revistas y editores de otros países de Europa, Australia y Nueva Zelanda, teniendo en común su experiencia en el proceso editorial y su dedicación preferente a temas generales de la medicina. El Comité se relaciona y colabora con otras organizaciones que tienen objetivos comunes o parcialmente similares, pero mantiene una estricta independencia para su organización y la elaboración de sus documentos. El número de miembros se mantuvo limitado, para facilitar sus discusiones y toma de decisiones. Dado que las primeras reuniones se realizaron en Vancouver, el Comité fue conocido también como "Grupo de Vancouver", pero luego variaron los sitios de reunión y adoptaron el nombre oficial de "International Committee of Medical Journal Editors (ICMJE)".

Un número creciente de revistas médicas se adscribieron a las recomendaciones del ICMJE. La Revista Médica de Chile, siendo Editor Jefe el Dr. Alejandro Goic G. y Editor Adjunto el Dr. Humberto Reyes B., comunicó a los autores de manuscritos y a los lectores que adoptaba estas recomendaciones y ello quedó explícito en las "Instrucciones a los Autores de Manuscritos". Fue reconocida por el ICMJE como revista adherente a los URM, desde su segunda edición, en $1982^{2}$. Actualmente los URM son aplicados por la mayoría de las revistas médicas del mundo, cualquiera sea el idioma en que se publican, y su cumplimiento es un elemento de juicio importante para las instituciones académicas y las sociedades científicas que juzgan a las revistas y a los manuscritos publicados. Más aún, para las bases de datos de literatura científica de corriente principal son un requisito indispensable para aceptar el ingreso y la permanencia de una revista.

Si bien el propósito inicial de los URM fue uniformar el formato de los manuscritos, en sus ediciones posteriores este propósito se ha ampliado (ver www.icmje.org). Actualmente incluyen: definiciones y requisitos de la autoría; las condiciones que debe cumplir la revisión por pares externos; las relaciones entre editores, autores y revisores de manuscritos; los derechos y deberes de los editores, y sus relaciones con las sociedades o entidades propietarias de las revistas; la necesidad de publicar estudios con resultados negativos; los principios éticos que deben cautelar las revistas médicas, que rigen la conducta e independencia de los investigadores, la protección de los seres humanos y los animales sujetos de investigación; un registro independiente de los ensayos clínicos, con acceso libre en Internet; la honestidad en los manuscritos y la declaración pública de potenciales conflictos de intereses; las normas que deben respetar las publicaciones electrónicas. La intención del ICMJE es "ayudar a los autores y editores en su tarea común de crear y difundir informes de estudios biomédicos que sean exactos, confiables, claros y fácilmente accesibles" (traducción libre del editorialista). La confiabilidad de la investigación, en cuanto puede afectar a la seguridad de los pacientes, requiere exigir la transparencia de la información proporcionada en cualquier manuscrito científico médico. Para ello se debe hacer accesible a los lectores toda información importante que subyace en los manuscritos.

En el año 2009, el ICMJE estaba integrado por los editores de doce revistas, más un representante de la National Library of Medicine (MEDLINE) y otro de la World Association of Medical Journal Editors (WAME), todos con voz y voto en sus decisiones corporativas. Ese año difundió en su página web y en notas en las revistas integrantes del Comité, su interés por incorporar dos revistas más. Podían postular revistas que, entre otros requisitos, procedieran de áreas geográficas no representadas por sus miembros, cuyos editores tuvieran experiencia en la función editorial y la ética de las publicaciones, ambos datos avalados por la historia de la revista, el curriculum vitae del editor respectivo y el respaldo oficial de la institución responsable de su publicación. La Sociedad Médica de Santiago-Sociedad Chilena de Medicina Interna, representada por su Presidencia y luego ratificada por su Directorio, apoyó la postulación de la Revista Médica de Chile. El 9 de febrero de 2010, la Dra. Christine Laine, MD, MPH, Editora de Annals of Internal Medicine, en representación del ICMJE, nos informó que la Revista Médica de Chile había sido seleccionada para incorporarse como miembro de esa entidad y solicitó que su Editor Jefe asistiera a la reunión del Comité, a efectuarse del 12 al 14 de abril de 2010 en Queenstown, Nueva Zelanda. La otra revista incorporada al ICMJE es el Chinese Medical Journal (publicado en inglés), cuyo Editor Jefe Dr. Getu Zhaori es, entre otras funciones, miembro del "Editorial Board" de New England Journal of Medicine. En estas circunstancias, la Revista Médica de Chile es una de pocas integrantes del ICMJE que se publican en 


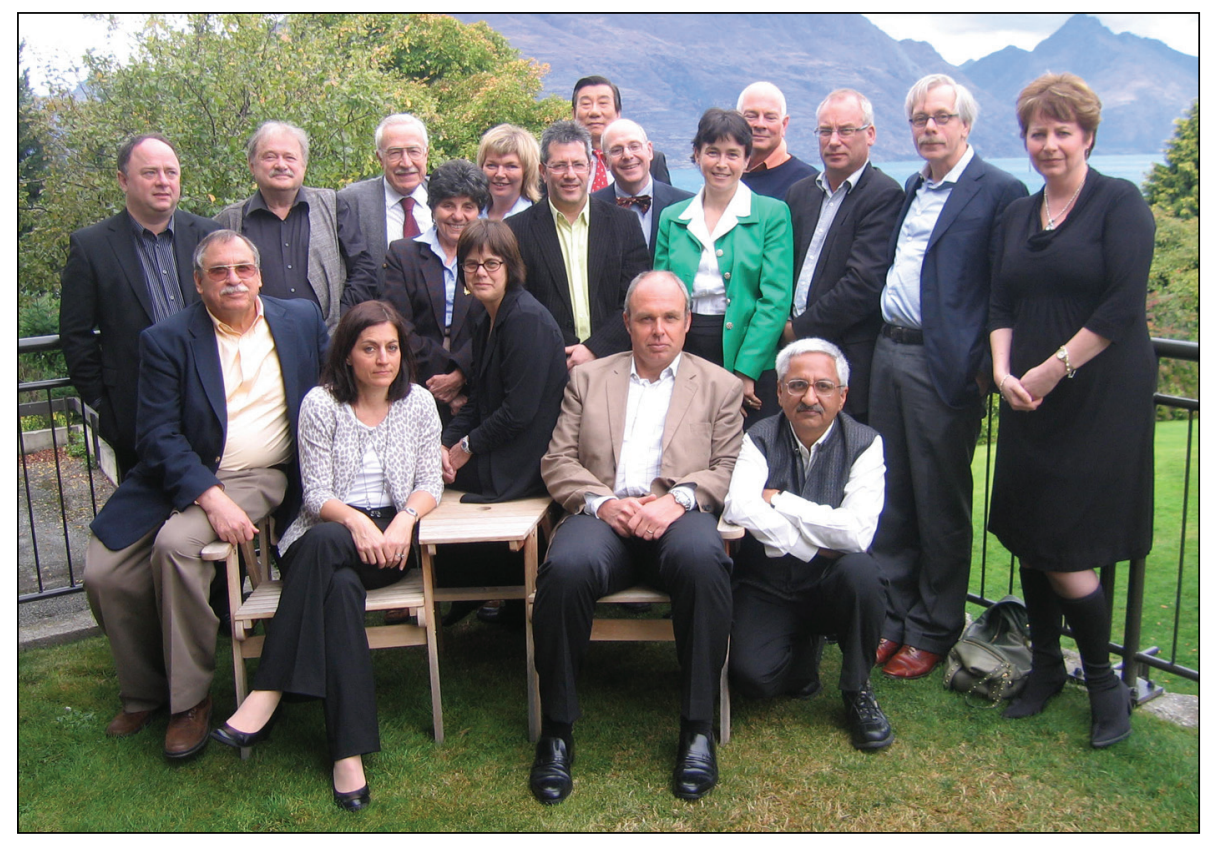

Asistentes a la reunión del /CMJE en Queenstown, Nueva Zelanda, del 12 al 14 de abril de 2010: De izquierda a derecha: Sentados: Sheldon Kotzin (Associate Director for Library Operations, National Library of Medicine, U.S.A.); Christine Laine (Editora, Annals of Internal Medicine); Cynthia Mulrow, Deputy Editor, Annals of Internal Medicine); Frank A. Frizelle (Editor, The New Zealand Medical Journal); Peush Sahni (Representante, World Association of Medical Journal Editors). En pie: Jacob Rosenburg (Journal of the Danish Medical Association); Martin B. Van Der Weiden (Editor, The Medical Journal of Australia); Humberto Reyes (Editor, Revista Médica de Chile); Catherine D. DeAngelis (Editora, Journal of the American Medical Association); Charlotte Haug (Editora, Norwegian Medical Journal); Paul C. Hébert (Editor, Canadian Medical Association Journal); Getu Zhaori (Editor, Chinese Medical Journal); Jeffrey M. Drazen (Editor, New England Journal of Medicine); Ana Marusic (Editora, Croatian Medical Journal); Tony Delamothe (Deputy Editor, British Medical Journal); Roger Mulder (Subeditor, The New Zealand Medical Journal); Peter W. De Leeuw (Editor, Nederlands Tijdschrift voor Geneeskunde - Dutch Journal of Medicine); Astrid James (Deputy Editor, The Lancet).

idioma no-inglés, la única cuyo idioma oficial es el español y la primera que ingresa desde el ámbito iberoamericano.

A la reunión en Queenstown asistieron todos los miembros del ICMJE o sus representantes (ver Foto). Se adoptaron acuerdos que serán publicados en la página web del ICMJE y reproducidos en sus revistas integrantes. Destacamos la intención de promover el uso de un "formulario uniforme para la declaración de potenciales conflictos de intereses", incluido en los URM desde octubre de $2009^{3}$. Este formulario debe ser llenado por cada uno de los autores de todo manuscrito que se someta a publicación, en cualquiera revista adscrita a los URM. En los manuscritos que se publiquen, los formularios respondidos por los autores se ponen a disposición de los lectores en la página web de la revista respectiva, permitiéndoles formarse un juicio personal sobre la influencia que pudieron tener en un manuscrito los potenciales conflictos de intereses declarados por sus autores.

El formulario inicial está disponible en la página web www.icmje.org y ya es exigido por algunas revistas médicas. Los lectores de la Revista Médica de Chile pueden ver cómo funciona en la realidad si eligen cualquier artículo publicado recientemente en New England Journal of Medicine o en Annals of Internal Medicine: en la versión electrónica de la revista, haciendo clic ("pinchar") en la opción "Disclosures" se consigue el acceso a los formularios llenados por cada uno de los autores de ese artículo.

Anticipándose a una eventual dificultad idiomática para comprender algunos términos del Formulario, en la reciente reunión del ICMJE se aprobó adjuntarle un "glosario" de términos 
técnicos en inglés y una traducción de las "Instrucciones" a los idiomas oficiales de la Organización Mundial de la Salud y de los miembros del ICMJE. El formulario en sí mismo sólo se ofrecerá en inglés, considerando que su propósito es proporcionar a los autores y lectores un documento con utilidad universal. La Revista Médica de Chile deberá adscribirse a esta normativa, que se suma a otros esfuerzos necesarios para cautelar la transparencia de las publicaciones médicas. Para aplicar el Formulario esperaremos que esté disponible su versión actualizada en 2010, con la traducción al español de las "Instrucciones".

El ingreso de la Revista Médica de Chile al International Committee of Medical Journal Editors implica nuestra incorporación a un grupo selecto de personas que recoge la experiencia de revistas de corriente principal y de entidades prestigiosas para proponer recomendaciones sobre cómo conducir las publicaciones médicas. Esta es una responsabilidad que implica la misión de ser, ahora como desde hace 138 años, más útiles a los lectores, proporcionándoles una vía importante para la difusión de nuevos conocimientos en medicina interna y otras especialidades médicas, y en las ciencias en general, confiando en que defenderemos la seguridad y el bienestar de los seres humanos a quienes atienden.

\section{Referencias}

1. International Steering Committee. Uniform Requirements for Manuscripts Submitted to Biomedical Journals. Ann Intern Med 1979; 90: 95-9.

2. International Committee of Medical Journal Editors. Uniform Requirements for Manuscripts Submitted to Biomedical Journals. Ann Intern Med 1982; 96 (Part 1): 766-71.

3. Drazen JM, Van Der Weiden MB, Sahni P, Rosenberg J, Marusic A, Laine C, et al. Uniform Format for Disclosure of Competing Interests in ICMJE Journals (Editorial). N Engl J Med 2009; 361: 1896-1897 [This editorial was published simultaneously in all ICMJE member journals]. 